\section{ESTUDIO Y CONCEPTOS SOBRE LA VIOLENCIA DURANTE EL NOVIAZGO*}

STUDY AND CONCEPTS ON DATING VIOLENCE
DURING

ESTUDO E CONCEITOS SOBRE A VIOLÊNCIA DURANTE O NAMORO

\section{RESUMEN}

La presente investigación es un aporte a los escasos estudios realizados sobre la violencia durante el noviazgo en Colombia. Para la comprensión de este fenómeno, en primer lugar, se hace alusión a los principales conceptos acerca del tipo y la naturaleza de la violencia, los factores de riesgo asociados y sus efectos, y a algunos estudios realizados al respecto. Luego, mediante la aplicación de la encuesta sobre el índice de severidad de la agresión y el cuestionario de violencia intrafamiliar, se realizó un estudio con 40 alumnas, entre los 14 y 17 años, de una institución educativa distrital en la localidad de Usme, Bogotá. Allí se halló que el 90\% de las estudiantes se encuentran en relaciones de abuso severo y violento, es decir, que requieren de una intervención urgente pues su vida y salud física y mental están en peligro. Asimismo, se detectaron factores de riesgo relacionados con la violencia intrafamiliar, el consumo de drogas y/o alcohol, los celos y el rol dominante del hombre en las relaciones de noviazgo.

\footnotetext{
* Esta investigación hace parte del proyecto de grado para la maestría en Asesoría Familiar y Gestión de Programas para la Familia, de la Universidad de la Sabana (Chía, Colombia), dirigida por la profesora Paola Akl de la misma institución.
}

a. Diana Bernal es licenciada en Educación Preescolar y docente del IED Gabriel García Márquez, y candidata a magíster en Asesoría Familiar y Gestión de Programas para la Familia en la Universidad de la Sabana.

b. Bibiana Hernández es licenciada en Pedagogía Reeducativa y docente de la IED Gabriel García Márquez, y es también candidata a magíster en Asesoría Familiar y Gestión de Programas para la Familia en la Universidad de la Sabana.

c. Paola Akl es psicóloga de la Universidad de la Sabana, magíster en Psicología de la Université Laval (Québec, Canadá), y docente de la Universidad de la Sabana.

Diana Yesmín Bernal Pinilla ${ }^{a}$ juanitabernal@hotmail.com Bibiana Patricia Hernández Cuchumbe bibi-juli@hotmail.com Paola María Akl Moanack ${ }^{c}$ paola.akl@unisabana.edu.co Fecha de recepción: 15 de febrero de 2016 Fecha de revisión: 19 de febrero de 2016 Fecha de aceptación: 3 de marzo de 2016

MISIÓN JURÍDICA

Revista de Derecho y Ciencias Sociales Bogotá, D.C. (Colombia) Colaboradores Externos Nacionales Núm. 11 Año 2016

Julio - Diciembre, pp. 273 - 287

ISSN 1794-600X 


\section{PALABRAS CLAVE}

Violencia en el noviazgo, tipos de violencia, naturaleza de la agresión, violencia sexual, violencia física, violencia psicológica, factores de riesgo, efectos de la violencia en el noviazgo.

\section{ABSTRACT}

This research is a contribution to the few studies on dating violence in Colombia. To understand this phenomenon, in the first place, it refers to the main concepts of the type and nature of violence, the associated risk factors and their effects, and to some research done on the subject. Then, by applying the survey of the severity aggression index and the questionnaire of domestic violence, a study was made involving 40 female students between 14 and 17 years old from a public educational institution in the locality of Usme, Bogota. This paper found that the $90 \%$ of the students are in a severe and violent relationship, it means that they require an urgent intervention because their life and physical and mental health are in danger. Moreover, risk factors associated with domestic violence, drugs and alcohol consumption, jealousy and the dominant role of men in dating relationships were identified.

\section{KEY WORDS}

Dating violence, types of violence, nature of aggression, sexual violence, physical violence, psychological violence, risk factors and effects of dating violence.

\section{RESUMO}

Esta pesquisa é uma contribuição para os poucos estudos sobre violência durante o namoro na Colômbia. Para entender esse fenômeno, em primeiro lugar, é feita referência aos principais conceitos sobre o tipo e a natureza da violência, fatores de risco associados e os seus efeitos, além de alguns estudos realizados. Então, através da aplicação do inquérito sobre o índice de gravidade da agressão e questionário violência doméstica, foi realizado um estudo com 40 estudantes entre 14 e 17 anos de uma escola distrital em Usme, Bogotá. Lá se verificou que $90 \%$ dos alunos têm relações de abuso grave e violento, ou seja, que exigem intervenção urgente porque sua vida e saúde física e mental estão em perigo. Além disso, fatores de risco relacionados à violência doméstica, o consumo de drogas ou álcool, o ciúme e o papel dominante dos homens no namoro foram detectados.

\section{PALAVRAS-CHAVE}

Violência no namoro, tipos de violência, a natureza da agressão, violência sexual, violência física, violência psicológica, fatores de risco, os efeitos da violência no namoro.

\section{INTRODUCCIÓN}

Cuatro datos relevantes sobre el informe del Instituto Nacional de Medicina Legal y Ciencias Forenses (INMLCF) para el año 2014: de los 48 849 casos presentados de violencia contra la pareja, la mujer fue la principal víctima $(85 \%$ de los reportes); la población etaria con una mayor frecuencia en el ejercicio de actos de violencia fue la comprendida entre los 15 y 29 años (el 52\% de los casos); los mayores agresores, sin distingo de género, fueron los compañeros permanentes (46\%), excompañeros permanentes (27\%), esposos (13\%) y novios y exnovios (8\%); por último, las principales razones detectadas para llevar a cabo estos actos de violencia fueron la intolerancia, los celos y la drogadicción y el alcoholismo (2015, pp. 209-213). Lo anterior evidencia, por un lado, que los actos de maltrato en el seno de la vida en pareja son un asunto de interés general y, en consecuencia, deben ser tratados, desde la perspectiva de la intervención estatal, como una problemática social y de salud pública. Pero también, por el otro lado, que para una comprensión integral del fenómeno se requiere de un abordaje que involucre diversas variables que no solo están relacionadas con el género (el dato más disiente de la estadística), la edad de las víctimas y los victimarios, el tipo de relación sentimental y las motivaciones que detonan la violencia contra la pareja, sino también con el contexto familiar, social, económico y cultural en el que se desenvuelven los individuos que deciden entablar una relación amorosa. Datos, sin embargo, no discriminados en el informe del INMLCF.

No es objeto de la presente investigación realizar un estudio exhaustivo de cada una de las variables que intervienen y explican el fenómeno de la violencia en pareja; por el contrario, aquí nos enfocaremos, primero, en una arista de la problemática que no ha sido lo suficientemente estudiada, y, segundo, en otra que nos permitirá caracterizar a la población y conocer, según el entorno, cuáles son los factores que más inciden en este comportamiento violento. Conceptualmente es posible diferenciar dos tipos de relaciones en pareja: Una, aquellas personas que mantienen y conviven en 
un mismo espacio, esto es, matrimonios o uniones maritales de hecho y, otra, aquellas que simplemente establecen un vínculo afectivo, o mejor, amoroso, sin que ello implique la convivencia en un solo espacio; se trata pues del noviazgo. Decimos que este tipo de parejas no ha sido objeto de suficientes análisis en el país, en tanto que ha sido la primera forma de relación interpersonal a la que mayor atención se le ha prestado. El concepto de violencia intrafamiliar es un buen ejemplo de la tendencia en los estudios: al remitirnos al concepto de familia es automática la referencia a un núcleo conformado por el esposo, esposa, compañera o compañero, padre, madre e hijos; es decir, necesariamente nos ubica en una relación marital o de convivencia. Lo cual, empero, deja de lado una forma anterior a la vida en familia: de nuevo, el noviazgo. Es por este motivo que el estudio pretende realizar un análisis, con esta población objetivo y un contexto determinado, sobre la presencia de la violencia y los factores que inciden en la pareja para ejercerla.

Las cifras citadas sustentan que la violencia durante el noviazgo nutre una buena parte de las estadísticas en el país. Que quienes cometan estos actos o sean víctimas de ellos hagan parte de una población entre los 15 a 29 años, edad en la que comúnmente se entablan este tipo relaciones, es un indicio de que la agresión comienza desde temprana edad en la forma de relacionamiento que es base para la posterior constitución de una familia. Es también cierto que en la relación en pareja una de las partes es más vulnerable que otra o, por lo menos, ha sido victimizada con mayor frecuencia: la mujer. De ahí que en el estudio se hayan elegido a las mujeres adolescentes; lo cual no quiere decir que la violencia no se ejerza desde la otra parte, es decir de la mujer hacia el nombre. El enfocarnos en la desagregación de la población, primero por tipo de relación y luego por género, obedece a los criterios de mayor vulnerabilidad (adolescentes y mujeres), y a la intención de incentivar que otras investigaciones aborden el fenómeno desde otros criterios.

Dijimos asimismo que una mirada integral al fenómeno debe abarcar aspectos tales como el entorno familiar, socio-económico y cultural. De su abordaje, luego, depende la identificación de factores de riesgo -objeto previamente de maltratos por parte de uno o varios integrantes de la familia, conocidos que ejercen o han ejercido la violencia en ambientes comunitarios o escolares, existencia de patrones culturales que legitiman la violencia, etcétera - que precipitan o contribuyen a que estas agresiones se den. No solo se trata de identificar los riesgos asociados al contexto con el ánimo de prevenirlos tempranamente, también de entender que, como en un ciclo, la víctima del noviazgo puede ser la futura víctima del matrimonio.

Así las cosas, en la primera parte de la investigación se mencionan los principales conceptos para entender el noviazgo y violencia de pareja, las categorías de la agresión, los factores y los efectos de su prevalencia, y las discusiones que han suscitado los estudios sobre estas agresiones. Seguidamente, se hace una breve descripción de la metodología empleada y la forma de obtención de la información, de la muestra elegida y el tipo de relaciones estadísticas empleadas. Tercero, se hace un análisis de los resultados obtenidos y se termina con algunas conclusiones y recomendaciones sobre la violencia durante el noviazgo.

\section{Sobre el concepto de violencia}

Una buena forma de aproximarse al análisis del fenómeno de la violencia puede hallarse en el Informe mundial sobre la violencia y la salud de la Organización Mundial de la Salud (OMS) del año 2002. Allí, encontramos desde una definición integral del concepto, su categorización, causas, efectos y dinámicas, hasta las estrategias y recomendaciones, individuales y colectivas, como sociedad y como Estado, para entender y prevenir su práctica. Partir de la conceptualización de este informe de la OMS nos permitirá abordar, al tiempo que organizar el debate comenzado por sus aspectos más generales para luego sí ahondar en el tipo de violencia que es objeto del presente estudio.

Para la OMS la violencia debe abordarse desde la perspectiva de la salud pública, esto es la preocupación por la salud de las comunidades y las poblaciones, con un enfoque especial en los grupos más vulnerables o cuya salud corre mayor riesgo o amenaza. En aras de conservar, promover y mejorar la salud, el proceso sugerido para alcanzar estos objetivos es la definición y consideración de la magnitud del problema, la identificación de sus causas, los posibles caminos para afrontarla, y la aplicación, con base en evidencia científica sólida, de las medidas para la prevención, promoción y mejora de la salud pública (2002, p. 4). En su orden de mención, la violencia, según la OMS, es:

El uso deliberativo de la fuerza física o el poder, ya sea en grado de amenaza o efectiva, 
contra uno mismo, otra persona o un grupo una comunidad, que cause o tenga muchas probabilidades de causar lesiones, muerte, daños psicológicos, trastornos del desarrollo o privaciones (2002, p. 4).

Esta definición trae a colación varios aspectos que sustentan la intervención estatal desde la óptica de la salud pública. Primero, alude a un comportamiento intencional, es decir, que existe un proceso premeditado para utilizar la fuerza o el poder para derivar de ello, por ejemplo, algún comportamiento de la persona a la que se agrede. A este respecto, Enrique Chaux realiza una diferenciación entre la agresión reactiva, que es aquella que ocurre como respuesta a una ofensa ya sea real o percibida, y la agresión instrumental que, por el contrario, no es precedida por una ofensa sino más bien el uso de la fuerza como instrumento para lograr un objetivo (2003 a, p. 49). Sea cual sea el tipo de agresión, queda claro que detrás de la acción violenta es dable hallar una motivación, un causa que la activa. De ahí la expresión "uso deliberativo de la fuerza o del poder" y que, en consecuencia, excluye otras manifestaciones que tienen que ver, por ejemplo, con trastornos o patologías que le impiden al individuo actuar de manera deliberada.

En segundo lugar, diferencia los tipos de agresión que se pueden generar: desde la amenaza hasta la concreción de la misma. Desagregadamente, la agresión ${ }^{1}$ puede ser de naturaleza psicológica, en donde se busca herir a través de la palabra o los actos -v. gr. insultos, amenazas, descalificaciones o humillaciones -; física, en donde ocurre el daño físico a la persona objeto de la agresión; y sexual, en donde el contacto sexual es no consentido (Barrientos, Molina y Salinas, 2013 a, p. 101). De los tres tipos agresión, sin embargo, es la de naturaleza sexual la que tiene una connotación más degradante pues en ella pueden confluir la agresión física

1.Enel presente texto se toman como referencia los tres tipos de agresión - psicológica, física y sexual. Empero, varios autores hacen referencia a otros tipos de agresión o de violencia como lo son la relacional (afecta las relaciones de familia y de amigos), la económica y la verbal (Chaux, 2002 a; Olvera, Arias y Amador, 2012). Pese a que cada una aporta más características a la naturaleza de la agresión, la violencia psicológica, si la definimos de forma extensa, puede abarcar cada una de ellas. Por ejemplo, siguiendo a González (2009), esta agresión es una conducto que busca desvalorizar a la parejay se manifiesta, entre otras más, en las siguientes actitudes: amenazas o insultos, humillaciones, aislamiento social y económico, celos, posesividad, destrucción de objetos personales o abandono (p. 29). Definición que engloba la particularidad de la agresión verbal, económica o relacional. (golpes o maltratos para doblegar a la víctima) y la psicológica (ofensas y expresiones que maltratan o humillan a la víctima). Se trata así de formas de manifestación de la violencia que se ejercen sobre la persona para conseguir un fin determinado o bien ratificar relaciones de poder de uno sobre otro.

Tercero, clasifica a las personas, comunidades o poblaciones que son objeto de agresión. De acuerdo con la OMS, existen tres categorías. La agresión autoinfligida o dirigida contra uno mismo se entiende como la tentativa de hacerse daño, causarse lesiones o mutilaciones. La agresión interpersonal que a su vez se subdivide en violencia intrafamiliar y/o de pareja ${ }^{2}$ y violencia comunitaria. Finalmente, la agresión colectiva y que está más relacionada con la violencia instrumental puesto que se ejerce "(...) frente a otro grupo de individuos, con objeto de lograr objetivos políticos, económicos o sociales" (OMS, 2002, p. 6). De este mapa de la violencia, empero, será objeto de análisis la segunda clasificación y más específicamente, aquella derivada de la violencia en pareja. Lo cual no significa que se eluda su referencia en esta investigación ya que como ha demostrado Chaux la violencia común sí puede tener una relación indirecta con el conflicto armado porque, entre otros factores, en los ambientes en donde es recurrente la violencia, se facilitan los aprendizajes sobre la efectividad y legitimidad de la agresión (2002, p. 52). Pero también en razón a que los efectos de la violencia sobre la víctima pueden conducir, como se verá, a intentos de suicidio, consumo de sustancias o trastornos de la alimentación.

Por último, precisamente con respecto a los efectos o secuelas ocasionados con el uso de la fuerza o el poder, la OMS incluye la muerte de la persona objeto de agresión, hasta lesiones y trastornos físicos o psicológicos transitorios o permanentes. Es pues la conjugación de todos estos elementos para definir la violencia los que justifican la intervención desde la perspectiva de la salud pública puesto que, por un lado, involucran, como un todo, al individuo, sus relaciones personales y a la sociedad y, por el

2. Para efectos de esta investigación, el concepto de relaciones de pareja, según Straus (como se citó en González, 2008, p. 43), se remite a aquellos "... encuentros para la interacción social y actividades compartidas con una explícita o implícita intención de continuar la relación hasta que una de las dos partes la acaba o hasta que se establece alguna otra relación más comprometida". De esta definición se deduce que los encuentros ocasionales no entran dentro de la categoría relaciones de pareja. 
otro, tienen un impacto en las esferas económicas, sociales, culturales y judiciales.

En ese sentido, la OMS afirma que para una comprensión integral del fenómeno de la violencia se debe atender a su complejidad, a su naturaleza multifacética: "La violencia es un fenómeno sumamente complejo que hunde sus raíces en la interacción de muchos factores biológicos, sociales, culturales, económicos y políticos" (OMS, 2002 , p. 10). A este respecto, conviene emplear la síntesis realizada por Salas (2005) para entender la interacción de estos factores que no son otra cosa sino las causas y el origen de la violencia. Haciendo énfasis en los detonantes de la agresión intrafamiliar, Salas agrupa estos factores en tres grandes grupos: individual, familiar y social o comunitario (2005, p. 4). En el primero, Salas cita los aspectos genéticos y biológicos, además de otras circunstancias como la situación laboral, la condición socioeconómica, el consumo de sustancias como el alcohol y las drogas, y el haber sido testigo o víctima de la violencia en el hogar. En el segundo grupo se encuentran factores relacionados con las características del hogar (p. ej. pobreza, hacinamiento, ingresos) y con sus dinámicas: “(..) el rol de género en la familia, las relaciones de poder entre los miembros del hogar, si las normas son autoritarias o igualitarias, etc." (2005, p. 4). Lo social o comunitario, por su parte, está ligado al entorno violento en la sociedad, la desigualdad en los ingresos, la precariedad de las instituciones encargadas del control y las prácticas o normas culturales (2005, p 5). Esta distinción, aclara Salas, hace parte de un conjunto de investigaciones denominadas modelos ecológicos de la violencia (2005, p 5). Es justamente este modelo el que sugiere y adopta la OMS, por cuanto "su principal utilidad estriba en que ayuda a distinguir entre los innumerables factores que influyen en la violencia, al tiempo que proporcionan un marco para comprender cómo interactúan" (OMS, 2002, p. 11).

Los innumerables factores los agrupa en los aspectos biológicos y personales, las relaciones (incluye además de las familiares las generadas en el entorno social: amigos, compañeros o parejas), y el entorno comunitario y la estructura social. Tener en cuenta esta visión no solamente cumple con el enfoque holístico de la intervención en salud pública, sino que también contribuye al esclarecimiento de las causas y las medidas a tomar, desde los distintos niveles o factores de violencia identificados, para prevenir esta práctica.
Ahora bien, teniendo en cuenta esta breve conceptualización y tipologías, a continuación ahondaremos en una de las tres categorías de violencia según la persona o población que la ejerce o la sufre: agresión interpersonal $y$, particularmente, la agresión entre parejas.

\section{La violencia en el noviazgo}

De las subdivisiones realizadas por la OMS de la violencia interpersonal, la intrafamiliar ha sido a la que mayor atención se ha prestado por parte de la investigación especializada. La violencia en pareja o durante el noviazgo, explica César Rey (2008 a), ha sido catalogada como insignificante o inusual, pero la evidencia fue demostrando que es un fenómeno más común de los que se pensaba.

Una de las razones por las cuales la investigación se ha concentrado en la comprensión de la violencia intrafamiliar, es la importancia que desde los mismos Estados se le da al concepto de familia. Así, por ejemplo, en la Constitución Política de Colombia se estipula taxativamente que la familia es la institución básica de la sociedad (art. $5^{\circ}$ ) y se regula el tipo de comportamiento y trato - "Las relaciones familiares se basan en la igualdad de derechos y deberes de pareja y en el respeto recíproco entre todos sus integrantes. Cualquier forma de violencia se considera destructiva de su armonía y unidad, y será sancionada conforme a la ley" (art. 42.ํ). Que sea considerada como el núcleo esencial de la sociedad implica, y al tiempo justifica, que el Estado dé un mayor énfasis para su conservación, protección y promoción.

Pese a ello, y aquí una segunda razón, las cifras citadas del INMLCF no se compadecen con su importancia. La violencia intrafamiliar sigue siendo una de las principales causas de muerte y de episodios de agresión en el país. A esto debe sumarse el hecho de que las víctimas generalmente son las personas más vulnerables dentro de la relación: las mujeres, los niños y los ancianos. Esta vulnerabilidad ha dado pie a una serie de medidas legales que los protegen especialmente y a una buena parte de la creciente literatura sobre la violencia de género, la infancia y adolescencia, y la tercera edad. El vacío, luego, radica en el estudio de una forma de interacción anterior al matrimonio y a la convivencia pero que es fundamental pues se trata de los intentos iniciales por entablar una relación afectiva que puede concluir en la conformación de una familia. 
La violencia intrafamiliar "ocurre entre individuos pertenecientes a un mismo núcleo familiar, con una relación matrimonial o de consanguinidad establecida" (Barrientos et. al., 2013 a, pp. 100-101). Por otra parte, la violencia en pareja sucede entre "las parejas de novios o de otra índole en donde existe una relación afectiva y sexual" (Rey, 2008 a, p. 228). En ambos casos, el uso de la violencia consiste en agredir, física, sexual o psicológicamente, al otro con fines tales como el control y dominio de la relación, modificación de conductas, entre otros. No obstante, la distinción entre ambas formas de asociación y de violencia es primordial ya que, siguiendo con Rey, existen dos diferencias clave con la violencia matrimonial:

a) La edad de los agresores y víctimas es ostensiblemente menor que la de parejas casadas, ubicándose en la adolescencia y adultez temprana, y b) las razones por las cuales se presentan y continúan las agresiones parecen ser distintas a las de la violencia conyugal, ya que no existe de por medio una responsabilidad paternal, contractual $o$ dependencia económica (p. 229).

Se resaltan de estas definiciones aquellos aspectos relacionados con la etapa en la que generalmente se da el noviazgo y el tipo de relación afectiva y sexual que se contrae. Según María González,

La adolescencia y/o juventud es un momento evolutivo de transición de la infancia a la madurez en el que se producen una gran cantidad de cambios afectivos, corporales y de valores, convirtiéndose en un periodo de especial vulnerabilidad y proclive al desarrollo de conductas desviadas (2009, p. 33).

Para Gisela Velásquez (2011),

La adolescencia es sin duda una etapa de la vida muy definitoria en cuanto a comenzar a perfilar las aspiraciones y la identidad personal, y en lo que se refiere a la condición propiamente emocional, en ese lapso vital -sin distinción étnica o de condición social- la inestabilidad es un rasgo permanente, y esa circunstancia suele ser muy propicia para las crisis recurrentes, $y$ si no se tienen el auxilio de personas cercanas o de profesionales pueden desbordarse lo mismo hacia el ámbito de las agresiones verbales, físicas, o buscar en las drogas y en el alcohol vías para escapar de esas realidades (p. 40).
El noviazgo, por el otro lado, es entendido como:

la vivencia amorosa con un valor subjetivo, que permite el conocimiento de la otra persona y el intercambio recíproco de valores que hacen parte de su ideal de vida romántica como lo son la tolerancia, confianza, ternura, respeto, sinceridad, pasión, comprensión, comunicación, autoestima... (Cruz, Lettau y Montoya, 2013 b, p. 192).

Mas a la par del intercambio recíproco de valores, aclaran Cruz et. al., la vivencia amorosa desencadena sentimientos negativos como "la desconfianza, celos, tristeza, agresividad, y desatención...” (2013 b, p. 192) que pueden terminar en actos de violencia contra la pareja.

Con estas definiciones se denota el grado de vulnerabilidad al que están expuestos los adolescentes y jóvenes pues la etapa está marcada por diversos cambios, definición de las aspiraciones y la identidad, sentimientos positivos y negativos con respecto al otro, idealizaciones del amor y de la vida en pareja, iniciación de la sexualidad, entre otros. Y en adición, afirma Rey (2008), los factores de riesgo para la violencia en pareja pueden ser mayores ya que “... los adolescentes no están suficientemente preparados para responder a los problemas que se presentan en las relaciones románticas" (p. 229).

Esta vulnerabilidad, reiteramos, se hace tangible al acudir a las cifras del INMLCF: el $52 \%$ de los agresores en este tipo de violencia son adolescentes y jóvenes entre las edades de los 15 a los 29 años, y el 85\% de las víctimas fueron mujeres. Esto pone de presente no solamente que la violencia de pareja es una problemática de salud pública, sino también que es la mujer a la que mayor énfasis se le debe dar para la prevención y garantía de los derechos vulnerados. El género de la víctima resulta pues una vulnerabilidad adicional.

Como colofón de lo anterior, tenemos una situación en la que se percibe una triple vulneración: por ser adolescentes, por ser novios y por ser mujeres.

De esta situación, como se acotó anteriormente, no se puede derivar que la violencia proviene exclusivamente de los hombres. Ese 15\% restante del total de las agresiones detectadas por parte de 
las mujeres del país en el 2014 debe ser también materia de estudio. Cuáles son, por ejemplo, los tipos de agresión más frecuentes, si responden a reacciones o actos planificados, o si existen variables diferenciadores que explican la violencia de la mujer contra el hombre, son algunas de las preguntas que pueden ser abordadas en este tipo de estudios. No obstante, para efectos de la presente investigación, nos ampararemos en las cifras de Medicina Legal y en la triple vulneración para justificar la población objeto de estudio.

Ahora bien, dichas vulneraciones propias de la etapa, se incluyen, atendiendo a la aproximación ecologista, dentro de ese conjunto de variables reseñadas y organizadas según las esferas individual, familiar y social o comunitaria, y el tipo de agresión presentada. En Colombia las investigaciones son muy escasas sobre los factores que precipitan la violencia entre parejas. Bexy Cruz et. al. investigaron la percepción sobre la violencia en el noviazgo, en especial, aquella relacionada con la violencia de género a 307 de estudiantes de la ciudad de Cartagena. De los factores de riesgo identificados el que mayor incidencia tuvo fue el social o comunitario. En los noviazgos de estos jóvenes "las actitudes violentas no se generan espontáneamente, sino que responden a estructuras culturales e históricas de relacionamiento desigual entre mujeres y hombre" (2013 b, p. 198). Las normas culturales acerca del rol del hombre y la mujer, refuerzan en estos estudiantes los estereotipos, al mismo tiempo que funcionan como justificadores de la violencia: "Las mujeres identifican a los hombres como personas fuertes y protectoras (...)

El prototipo de súper hombre agresor es aceptado (...) quienes en su posición de novias adoptan un rol pasivo aceptando los abusos que sobre ellas se generen" (2013 b, p. 194). En contraste, los hombres identifican a las mujeres "como el sexo débil, sumiso y resignado, con personalidad conflictiva posesiva y celosa" (2013 b, p. 194).

En cuanto a la naturaleza de las agresiones, la violencia psicológica, manifestada principalmente en actos verbales, fue la de mayor frecuencia entre los jóvenes. Intimidación y manipulación del otro, por ejemplo, al controlar su forma de vestir, restricción o control de amistades, las llamadas y forma de vestir (2013 b, pp. 194-195). La violencia de tipo físico, por otra parte, aunque menos constante, son aceptadas y naturalizadas por los participantes: "pellizcos, estrujones, miradas de reproche, y bofetadas, en espacios públicos y privados" (2013 b, p. 196). Son pues, explican las investigadoras, actos justificados por los celos, la pérdida de control, sentimientos de ira o furia o por simple reacción, defensa ante una situación percibida como hostil.

Otro estudio, en esta ocasión centrada en la violencia intrafamiliar, con 2295 mujeres entrevistadas, realizado por Luz Salas, evidenció los factores a través de los cuales la violencia se transmite de una generación a otra. Citamos este estudio porque aborda algunas variables que se relacionan con el fenómeno de la violencia entre parejas y en tanto que nos permite introducir el concepto de ciclo de la violencia. De acuerdo con Enrique Chaux dos fenómenos confluyen en la transmisión generacional o reproducción de la violencia. Los niños que viven en un ambiente violento observan una mayor probabilidad de replicar estos comportamientos y los niños que desarrollan estos comportamientos agresivos, tienden a mantener este comportamiento en el desarrollo de sus vidas. "Uniendo estos dos hechos se genera el ciclo de violencia" (2003 a, p. 48). Para nuestro caso, el ciclo de violencia es transversal a las variables que precipitan la violencia durante el noviazgo. Ante todo debe tenerse en cuenta los factores de riesgo relacionados con la dimensión individual, esto es: haber sido víctima o testigo de violencia en el hogar; el entorno familiar, a saber: relaciones de poder, normas autoritarias o el rol de género, etcétera; y el entorno social o comunitario, reflejado, por ejemplo, en los contextos violentos en los que se convive. Estos factores pueden evidenciarse en dos etapas: "Una característica encontrada constantemente, tanto en víctimas como victimarios de violencia en pareja, es la experiencia de haber presenciado o haber sido víctima de violencia en la familia de origen" (Rey, 2008, p. 234); por otra parte: "Se ha demostrado que la violencia durante el noviazgo puede ser un precursor de la violencia durante la vida marital" (Allen, Chávez, Lazcano, Rivera y Rodríguez, 2006, p. 289). Luego uniendo estas dos afirmaciones se genera el ciclo de la violencia en las parejas.

Volviendo al estudio de Salas, "la violencia intrafamiliar se transmite de generación en generación por aprendizaje - experiencias vividas en el hogar de origen" (2005, p. 38). Para romper con este ciclo de violencia, particularmente hacia la mujer, el estudio evidenció que el divorcio es uno 
de los mecanismos protectores y que coadyuvan a la transmisión de la violencia. El descenso porcentual, en estos hogares, es del $15.1 \%$. Aunado a ello, complementa salas, la intervención estatal mediante la implementación de políticas para su reducción y prevención resulta indispensable. Las variables socioeconómicas que pueden incidir en el origen de la agresión, disminuyen en los casos en donde la mujer obtiene empleo, tiene cobertura de servicios de salud y un mayor nivel educativo. De igual manera, la investigadora menciona que las políticas de control de natalidad, los programas de atención y prevención a las víctimas del maltrato familiar, las campañas de reducción de las drogas y el alcohol, influyen positivamente en la disminución de los índices de agresión severa contra la mujer (2008, pp. 38-39).

En el ámbito internacional, el estudio de Allen et. al., aplicado a los estudiantes de instituciones educativas públicas de Morelos (México), encontró que el tipo de agresión más empleado, en contravía de los resultados de la investigación de Bexy Cruz et. al., es la violencia física (empujones), seguido de la violencia psicológica (falta de respeto, humillación, improperios, etc.). Evidenció asimismo que los episodios de violencia en el noviazgo están asociados al consumo de cigarrillos y alcohol, el rendimiento académico y las relaciones sexuales. La prevalencia de la violencia es mayor en las consumidoras habituales, las de bajo rendimiento académico y en quienes ya han iniciado su vida sexual (2006, pp. 291-293).

Olvera et. al. (2012), llevaron a cabo una investigación con 100 estudiantes en un centro universitario de Zumpango (Estado de México) para identificar la presencia de violencia entre las parejas de novios. El $92 \%$ de las participantes aceptó la presencia de violencia psicológica por parte de sus parejas, y el $60 \%$ de ellas reconoció la existencia de agresión económica -derroche de dinero sin explicaciones sobre la distribución de los gastos, disposición de los recursos de la mujer, etc. (p. 161). La violencia física se evidenció con los maltratos leves: empujones, $40 \%$; besos a la fuerza, $15 \%$; mordidas o chupetones, $20 \%$; mientras que solo un $2 \%$ reconoció la agresión con golpes fuertes. Sobre la violencia sexual, finalmente, se registró que de la población activa sexualmente (casi el 50\%), el $72 \%$ ha vivido situaciones de sexo sin consentimiento.

César Rey hace un recorrido por los diferentes estudios realizados en Estados Unidos, Canadá,
México y Brasil con el ánimo de indagar acerca de cuáles son los factores de riesgo que mayor incidencia tienen en las agresiones durante el noviazgo. En la investigación de Sears (como se citó en Rey, 2008 a, p. 232) se encontró que los adolescentes varones que han ejercido los tres tipos de violencia manifestaban actitudes más conservadores frente a la mujer, justificaban la violencia en pareja, y tenían antecedentes, presenciales o directos, de violencia en la familia de origen. En cuanto a los factores ligados a los roles tradicionales de género, Rey ratifica la existencia de actitudes y patrones culturales en los hombres que facilitan o inducen a la agresión de su pareja. Sin embargo, acota, las diferencias entre hombre y mujeres que ejercen o han ejercido la violencia no es significativa en las investigaciones a las que alude en el artículo. La violencia "en las parejas jóvenes obedece, indistintamente del género, a un asunto instrumental, posiblemente mediado, en el caso de los varones, por una búsqueda de reafirmación de la masculinidad, y, en el caso de las mujeres, por una búsqueda de equidad" (2008 a, p. 233). De ahí que Rey advierta la necesidad, como lo mencionamos más arriba, de llevar a cabo investigaciones sobre los factores culturales y contextuales que explican la violencia de la mujer contra el hombre durante las relaciones de noviazgo pues es recurrente, argumenta, pensar que las agresiones de la mujer son solamente reactivas y no instrumentales.

Por otra parte, comenta que la probabilidad de ejercer la violencia se aumenta cuando las parejas de novios asimilan la violencia como algo natural o posible en los casos en los que se ha presenciado o experimentado la violencia en la familia de origen o bien a partir del "... conocimiento de pares que han efectuado actos de esa naturaleza" (2008 a, p. 235). Por último, Rey alude a otros efectos que también están asociados a la violencia como lo son el uso y abuso de sustancias psicoactivas, las riñas, la conducta sexual, los embarazos, los intentos de suicidio y los problemas para el control del peso corporal. Factores, en síntesis, que deben ser analizados para la comprensión del fenómeno y el direccionamiento de las decisiones que se tomen para atacarlo y prevenirlo.

Estos estudios nos confirman, primero, la presencia de actos de violencia entre las parejas de novios; segundo, la prevalencia de la violencia contra la mujer; tercero, las dinámicas en las que se dan estos actos de violencia, así como los factores de riesgo que detonan estas actitudes 
agresivas; y, en cuarto lugar, las variaciones del tipo de agresiones, factores y víctimas que impiden establecer datos concluyentes al respecto. Por estas razones, uno de los propósitos de esta investigación es analizar la presencia de la violencia en las relaciones de noviazgo y la identificación de algunos factores de riesgo mencionados en un grupo de mujeres estudiantes de una institución educativa de la ciudad de Bogotá. Un segundo propósito, ya surtido en este acápite, es el de aportar en la clarificación de la discusión teórica y metodológica (como se verá en seguida) para continuar abriendo las puertas y que otras investigaciones se acerquen y nutran el entendimiento de este fenómeno. Precisamente, a continuación se relaciona y organiza el concepto de violencia entre parejas a partir de lo citado más arriba. No se trata, desde luego, de una única forma de presentar la información y decantar de manera gráfica los conceptos ligados a las agresiones en pareja; es solamente un mapa tentativo abierto a las modificaciones o precisiones. Del mapa, cabe aclarar, se mencionan los tres tipos de violencia pero se desarrolla únicamente la violencia interpersonal y de ella, la de pareja o noviazgo. Aunque la violencia intrafamiliar puede asemejarse en materia de la naturaleza de la agresión, preferimos mencionar solo los factores de riesgo para la violencia en relaciones de noviazgo puesto que existen diferencias con respecto a las relaciones intrafamiliares que no permitirían un solapamiento de los mismos. De igual modo, se dejan de lado los efectos, las consecuencias de la violencia, para concentrarnos en este estudio en los contextos o situaciones en las que se da. Queda abierta pues esta posibilidad.

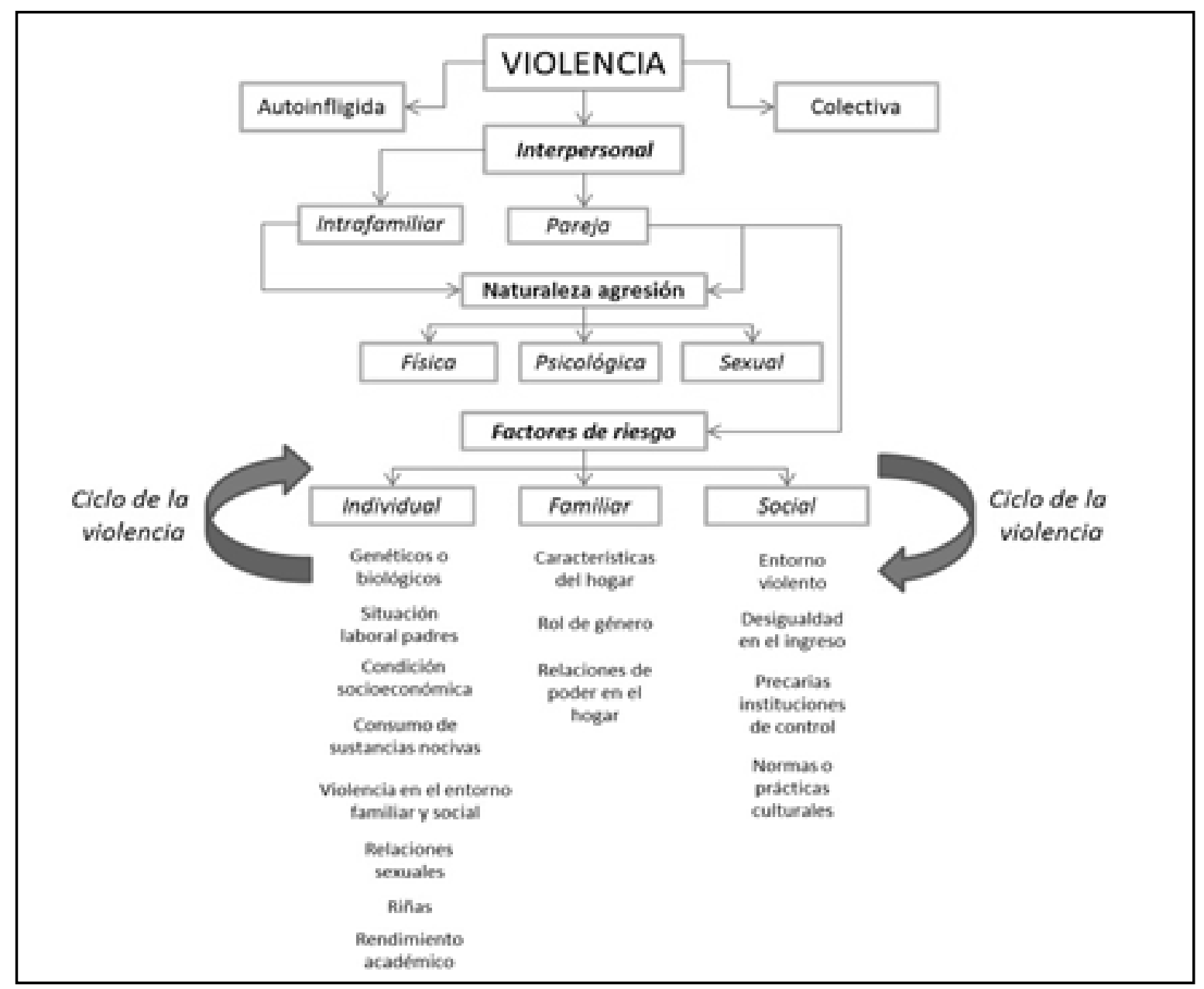

Figura 1. La Violencia durante el noviazgo. Fuente: elaboración propia. 


\section{METODOLOGÍA}

En aras de identificar la presencia de la violencia durante el noviazgo y analizar aquellos factores que la detonan, se realizaron dos encuestas a 40 estudiantes, de género femenino, del grado décimo de la Institución Educativa Distrital Gabriel García Márquez de la ciudad de Bogotá en la localidad de Usme. La forma de obtención de la información de estas estudiantes consistió en la aplicación, primero, de un cuestionario con 20 reactivos en una escala tipo Likert (frecuente, a veces, nunca) para detectar y medir el grado de violencia en el noviazgo, empleado por Bustamante, Gómez, Figueroa y Gómez (2008 b) ${ }^{3}$. La encuesta recaba información sobre la autoestima de las adolescentes a partir de la presencia de acciones dominantes que ejerce la pareja y que pueda desencadenar sentimientos de inferioridad, sumisión y conformismo; sobre el desarrollo de su sexualidad: cómo se relacionan el amor y el sexo, los actos de agresión con amor; o bien los comportamientos de aceptación por parte de las adolescentes en los casos de violencia entre parejas. Entre los reactivos de esta encuesta se encuentran las agresiones psicológicas hacia la mujer: apodos desagradables, burlas, control o vigilancia de su vida y acciones, temor, amenazas, presión para la consumir sustancias; las agresiones físicas: violencia causada por el consumo de alcohol o droga, golpes o lesiones que ameritan la intervención y/o atención de una autoridad; y las agresiones sexuales: presión para acceder a acceder a los deseos sexuales del novio, impedimento o condicionamiento para el uso de anticonceptivos o prácticas sexuales desagradables.

A partir de las respuestas de las adolescentes, la encuesta establece un índice de violencia (en la escala se asigna un puntaje de 2,1 y 0 ) que refleja el nivel de severidad de la agresión. De 0 a 5 puntos se trata de una relación sana, sin presencia de violencia, es decir, donde prima el respeto y los derechos de cada uno. De 6 a 15 puntos indica que hay unas señales iniciales de violencia en la pareja. De 16 a 25 puntos existe ya una relación de abuso pues cada vez más se emplea la violencia para resolver los conflictos. De 26 puntos a 40 la relación es de abuso severo: la violencia se da bajo cualquier pretexto y es frecuente e intensa. De 41 puntos en adelante la relación es violenta y requiere de medidas de seguridad y ayuda especializada.

3. El modelo de la encuesta puede ser consultada en el siguiente vínculo: http://es.slidesharenet/dulcegr/cuestionarioviolencia-en-el-noviazgo
A esta encuesta, que facilita la organización de la información según el índice de violencia, se le incluyó un segundo método de recolección de datos sobre los factores de riesgo que precipitan la violencia en el noviazgo. El Cuestionario VIF, elaborado por Akl y Docal (2014), está construido alrededor de 87 preguntas, agrupadas por 6 variables: (i) composición familiar y características socioeconómicas, (ii) situación económica familiar, (iii) convivencia en el hogar, (iv) experiencia migratoria, (v) participación en organizaciones sociales y (vi) violencia intrafamiliar y de pareja. De ellas, sin embargo, para el presente estudio solamente, pese a que se cuenta con la totalidad de las respuestas, se seleccionaron algunas preguntas de las variables, en su orden de mención, uno, dos, tres y seis pues estas apuntan directamente a los factores de riesgo relacionados con la dimensión individual, familiar y social. Para el análisis de las mismas, finalmente, se realizó un análisis estadístico descriptivo tomando como referencia la totalidad de la muestra y el índice de violencia detectado.

Ahora bien, la elección de la muestra mencionada obedece fundamentalmente a tres razones. La institución educativa Gabriel García Márquez, de carácter distrital, está ubicada en la localidad de Usme en la ciudad de Bogotá. Para el año 2013, el 7\% de los casos presentados de violencia intrafamiliar se dio en esta localidad: niños y adolescentes, 133 casos; entre familiares, 79 casos; entre parejas, 399 casos; $y$ contra los adultos mayores, 4 casos (Alcaldía Mayor de Bogotá, 2013 c). En otro estudio de Eliana Ortiz (2003 b), ya se señalaban algunos de los factores de riesgo más frecuentes detectados para la ocurrencia de la violencia intrafamiliar o de pareja en Usme:

(...) el bajo o el inexistente nivel educativo, no sólo en las víctimas sino también del grupo familiar y social en el que se presenta la problemática. Muchas de las víctimas no concluyeron sus estudios básicos, ni medios y un muy bajo índice de ellos lograron culminar estos ciclos escolares. Ello contribuye a que su situación de dependencia económica, social y emocional hacia el agresor sea permanente, encontrándose pocas posibilidades de romper el ciclo de violencia. Además en cuanto a la relación del agresor con la víctima, de un $100 \%$ de casos se evidencia que un $68 \%$ de estos casos, el agresor hace parte de la familia. El padre es un agresor importante en cuanto aparece como maltratante físico y emocional o ejerciendo violencia por abandono, negligencia o abuso sexual contra sus hijos. El mayor agresor sigue siendo el hombre; 
sin embargo las mujeres aumentan como agresoras psicológica y físicamente de sus hijos (sic) (p. 21).

La situación de violencia intrafamiliar y de pareja es pues evidente en esta localidad, lo cual justifica ahondar en los análisis sobre las agresiones durante el noviazgo.

La escuela, en segundo lugar, es un espacio idóneo para detectar la existencia de esta violencia, así como su severidad. Asimismo, es uno de los espacios institucionales esenciales para atacar y prevenir tempranamente las manifestaciones violentas de los adolescentes. De ellos, no obstante, se eligieron a 40 alumnas de grado décimo de bachillerato que tenían una relación sentimental y su edad oscilaba entre los 14 y los 17 años. Lo anterior bajo el supuesto de que en esta etapa se inician los noviazgos y las relaciones sexuales con la pareja. Finalmente, dos de las tres investigadoras hacen parte de esta institución educativa, lo que, de una parte, brindó cierta flexibilidad para que las estudiantes accedieran a responder las encuestas, $y$, de la otra, facilitará la toma de decisiones por parte de las directivas y docentes con el objeto de prevenir y erradicar estas conductas agresivas de los estudiantes.

\section{ANÁLISIS DE LOS RESULTADOS}

Con respecto a los resultados de la aplicación de la encuesta que permite evidenciar el grado de severidad de la violencia durante el noviazgo, los resultados son preocupantes. De las 40 alumnas, el $55 \%$, se encuentra inmersa en una relación violenta, es decir, que ya se sugiere una intervención urgente en razón a que su vida y salud física y mental se encuentran en peligro (en la tabla, valor 5). El 35\% está cerca de escalar a una relación violenta porque en sus relaciones sentimentales es frecuente acudir a la violencia (en la tabla, valor 4). El matiz aquí, en las relaciones de abuso severo, radica en que la pareja intenta remediar el acto una vez este lo ha ejecutado. Las relaciones de abuso y primeras señales de violencia fueron relativamente bajos: 8 y $2 \%$ respectivamente (en la tabla, valor 3 y 2); mientras que la inexistencia de violencia en la pareja fue nulo.



Gráfico No. 1. Fuente: elaboración propia.

El porcentaje de mujeres con índice severo de agresión es inverso a los resultados del estudio citado de Bustamante et. al. (2008 b): se "reveló que el $50 \%$ de las entrevistadas en las 5 universidades tiene los primeros indicios de violencia, el $12 \%$ una relación de abuso, el $5 \%$ abuso severo y el $2 \%$ una relación violenta (...)" (p. 35). Los resultados en nuestra investigación en lo referente a la naturaleza de la agresión, desagregado por nivel de severidad, arrojaron que del 55\% de las estudiantes con una relación severa, el $45 \%$ recibió una agresión de carácter psicológica, el 36\% de índole física y el restante sufrió agresiones sexuales. Del $41 \%$ de las alumnas con relaciones de abuso severo, $42 \%$ recibieron maltratos físicos, $41 \%$ fueron afectadas por la violencia psicológica y el $17 \%$ objeto de agresiones sexuales. De las estudiantes que se encuentran en una relación sentimental con visos de abuso o con primeras señales de violencia, la distribución, respectivamente, fue la siguiente: psicológica, 46\% y 55\%; física 8\% y 45\%; y sexual, 8\% y 0\%. 


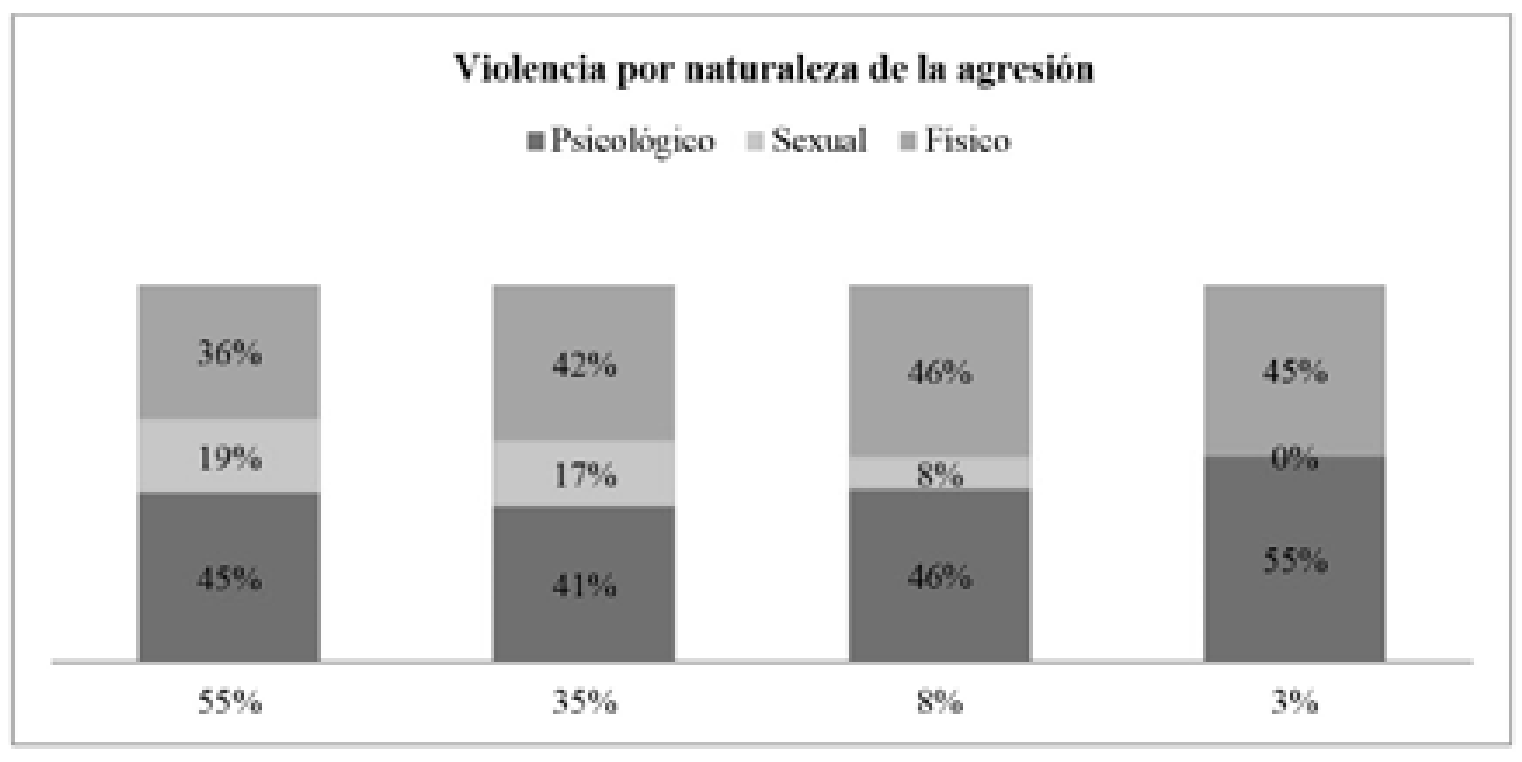

Gráfico No. 2. Fuente: elaboración propia.

El orden de la naturaleza de la agresión es similar a los estudios reseñados en el acápite anterior. La violencia psicológica continúa siendo la conducta más frecuente. Preocupa, sin embargo, la frecuencia de la violencia de carácter físico: desde la identificación de signos iniciales de violencia ya hay presencia de alguna forma de maltrato físico hacia la alumna. Pese a que la violencia sexual no es tan recurrente, se puede observar que, a medida que va aumentando la severidad de la agresión, esta es más común.

Lo anterior debe decantarse en la urgente implementación de estrategias que logren reducir desde ahora y en aras de romper con el ciclo de la violencia, estos índices de violencia hacia la mujer.

Por último, en lo atinente a la manifestación de estos tres tipos de agresión, se obtuvo, entre otras, que los novios frecuentemente (en un $38 \%$ ) y a veces (en un 53\%) quieren saber con quién anda y qué hace; frecuentemente (en un 23\%) y a veces (en un $50 \%$ ) se dirigen hacia ellas utilizando apodos o sobrenombres desagradables o groseros; frecuentemente (en un $35 \%$ ) y a veces (en un $28 \%$ ) se burlan de su cuerpo y exageran sus defectos en público y en privado; frecuentemente (en un $30 \%$ ) y a veces (en un 48\%) se sienten temerosas y tensas cuando están con él; frecuentemente (en un
$30 \%$ ) y a veces (en un 60\%) ignoran la opinión al momento de decidir qué hacer cuando salen juntos; frecuentemente (en un $25 \%$ ) y a veces (en un 63\%) han recibido golpes en algunas partes de su cuerpo; y, alarmantemente, con frecuencia (en un $20 \%$ ) y a veces (en un $40 \%$ ) les han causada lesiones que necesitan de atención médica, psicológica, jurídica y/o policial.

En cuanto a la aplicación del cuestionario VIF, la conformación de la familia de las estudiantes, desde la óptica de la esfera individual, no arrojó resultados significativos: el $64 \%$ pertenece a una familia nuclear completa, el $27 \%$ a una familia nuclear incompleta y el restante a una familia reconstituida. El nivel socioeconómico de todas ellas es bajo, es decir, debe oscilar entre los estratos 1 y 2 .

El nivel educativo de los padres, para la totalidad de la muestra, se distribuyó así: el $28 \%$ de las madres no completó la primaria; el $25 \%$ tiene la primaria completa; el $20 \%$ no culminó los estudios de bachillerato; el 13\% sí completo la educación secundaria; el 3\% no tiene ningún tipo de estudio; y tan solo el $10 \%$ tiene estudios técnicos, de educación superior completos o incompletos. Los padres, por el contrario, en un $30 \%$ tienen el bachillerato incompleto, el $23 \%$ la primaria sin terminar, el $20 \%$ con la educación 
secundaria terminada, y otro $15 \%$ con primaria finalizada. No obstante, ninguno de los padres continuó con la educación superior. Se ve entonces una diferencia, aunque leve, en formación académica básica. El 88\% de los hombres han cursado como mínimo la primaria y como máximo el bachillerato. El rango de la mujer con estos mínimos y máximos fue 11 puntos porcentuales menos. Ahora, si analizamos los datos a partir del índice de violencia, se destaca que el $82 \%$ de las madres cuyas hijas están en una relación violenta ninguna ha finalizado el bachillerato; mientras que este porcentaje se reduce al $73 \%$ en el caso de los padres. Para las adolescentes con abuso severo por parte del novio el nivel educativo es similar, aunque disminuye un poco: $57 \%$ para los padres y $64 \%$ para las madres. En general, el nivel educativo es bajo pues de los padres y madres tan solo el 6\% tiene un grado técnico y tecnológico culminado y solo dos de ellos han terminado estudios de educación superior.

En cuanto a la ocupación actual, el 60\% de los padres es, según la clasificación del cuestionario, obrero o empleado particular; mientras que las madres el $43 \%$ es ama de casa, $23 \%$ trabajan en el servicio doméstico y el $15 \%$ son obreras o empleadas particulares. Visto desde el índice de severidad de la violencia, en un promedio del $45 \%$ las madres de las niñas con relación violenta y de abuso severo son amas de casa. Sus padres, por otra parte, en un promedio de $59 \%$ son obreros o empleados particulares.

En el caso de la existencia de violencia en la familia de origen, las estudiantes afirmaron, en un $98 \%$, que no se considera víctima de violencia intrafamiliar. Ese porcentaje restante, corresponde a alumnas con relaciones de abuso severo en sus noviazgos. Esta afirmación se refuerza con un 95\% y $90 \%$ que aseguran que una persona que ha sido maltratada no se debe a que se lo haya buscado ni que la violencia sea un comportamiento normal para solucionar los conflictos.

Reconocen, en materia del entorno familiar y social, que algunos de los factores detonadores de violencia en la familia son la falta de recursos económicos $(53 \%)^{4}$, el consumo de drogas y bebidas alcohólicas (24\%) y los celos y la infidelidad (11\%). Se detectó además que en un $28 \%$ existen antecedentes de consumo de alcohol; en un 19\% consumo de sustancias psicoactivas; en un $17 \%$ denuncias de maltrato; en un 9\% celos y dependencia emocional. Se halló además que quien toma las decisiones importantes en el hogar es, en el 35\%, el padre y en $65 \%$ entre los dos. Lo cual se corresponde con la respuesta negativa, casi mayoritaria, a la pregunta sobre si el hombre debe dejar en claro que es él el que manda en el hogar.

De lo descrito, varias preguntas y sugerencias se deben mencionar. Primero, es evidente la existencia de maltrato hacia la mujer en estas primeras relaciones. Es importante anotar, que el hallar soluciones a la problemática es urgente e inaplazable ya que hay $90 \%$ de mujeres que sufren un maltrato severo. Esto, en segundo lugar, no se compagina completamente con los factores de riesgo analizados. Pese a las bajas condiciones económicas y educativas, no parece que esto tuviese una incidencia significativa en la violencia durante el noviazgo. Empero, es menester matizar el hecho de que el cuestionario fue respondido por las mismas alumnas y no por los padres. Quizás acudiendo a ellos y a otras fuentes de información estadística, se puede contar con mayor fiabilidad al momento de analizar los entornos pues los estudios citados sí muestran una fuerte incidencia de estos factores de riesgo. Es aquí relevante mencionar un aspecto que menciona Salas (2005): la promoción de la independencia de la mujer puede llevar a que cambien las relaciones de poder al interior del hogar y adquirir así una posición más equitativa en el mismo. Esa independencia, afirma,

(...) puede estar determinada por el aumento en el nivel educativo, mayores oportunidades laborales, mayores ingresos por trabajo para volverse valorada socialmente y por su compañero, para que él sienta la amenaza de que si la maltrata, ella tiene mayores posibilidades de rechazar el maltrato $y$ abandonarlo (p. 40).

En síntesis, otra forma de romper con ese círculo de la violencia. Lo mismo aplica para las mujeres en sus relaciones de noviazgo. Los datos confirman la sumisión de una frente al otro. Continuar con estudios de educación superior puede ser un factor que reduzca los niveles de

4. Esta pregunta, sin embargo, contrasta con la respuesta dada a otra pregunta similar y que indaga acerca de si la falta de dinero es responsable de la violencia intrafamiliar: un 80\% respondió que no y el otro 20 que sí. 
agresión hacia ellas, pero estos datos reflejan aún la prevalencia de una estructura social y cultural que no valoran el rol de la mujer en la sociedad.

También es de subrayar que por lo menos a nivel de percepción, las estudiantes rechazan la violencia como método para conseguir un fin y reconocen qué tipo de conductas facilitan la agresión en la pareja.

\section{CONCLUSIONES Y RECOMENDACIONES}

Resulta fundamental continuar ahondando en este tipo de estudios sobre la violencia durante el noviazgo; pero no solo teniendo en cuenta el enfoque de género sino también otras condiciones sexuales que pueden estar generando índices de violencia iguales o superiores. Es aquí donde las instituciones privadas o públicas deben apoyarse en este tipo de estudios para comprender el fenómeno y luego así formular políticas de prevención y reducción de los índices. Para ello, sin embargo, es vital incentivar los estudios para que desde la academia se construyan, refuercen o se utilicen metodologías, como la empleada en esta investigación, que hagan medible y entendible este tipo de prácticas. Sin ellas, la toma de decisiones se dificulta aún más y alienta la improvisación en las intervenciones que se hagan al respecto. Acudir a un enfoque ecológico es una buena manera de atender integralmente este grave problema de salud pública.

Hemos pretendido construir un marco teórico que facilite su comprensión pero consideramos que todavía hace falta bastante conceptualización y estudios que deriven directamente de la experiencia colombiana. Robustecer la identificación de los factores de riesgo en sus tres dimensiones y analizar los efectos de la violencia en pareja hacen parte de ese vacío que hay que ir llenando. Destacamos de lo escrito la aproximación desde las instituciones educativas porque es un espacio esencial en el desarrollo identitario, emocional y cognitivo de los adolescentes. Trabajar desde allí y no solo desde el núcleo familiar puede contribuir a la no transmisión de la violencia de generación en generación. Si bien en la escuela se replican los comportamientos aprendidos en el entorno familiar, asimismo es cierto que en ese espacio se construyen aptitudes y conductas de cara hacia la familia y la sociedad.

Se percibe en el estudio que a pesar de haber contestado las encuestas, aún existe mucho temor de delatar o denunciar los casos en los cuales la violencia hace su presencia ya sea por la dependencia económica y emocional o por que las consecuencias que se desencadenen por este motivo sean aún más graves. Los adolescentes por presentar altos niveles de inmadurez e inexperiencia, necesitan de orientación, guía y de alguna manera acompañamiento en el desarrollo del proceso emocional, para prevenir comportamiento violentos y dañinos no solamente a la pareja sino a sí mismos y a su entorno.

Se hace necesario así que el personal docente de los establecimientos educativos redunde en esfuerzos y acciones participativas en pro del bienestar del estudiante como parte integral dentro de un proceso, por ejemplo, de formación en valores ya que en una relación de noviazgo con características violentas no solamente se minimiza la dignidad de la persona agredida sino que también se deteriora y anula la calidad humana del agresor. Estrategias que se deben trabajar desde el campo de la educación como la interacción y el conocimiento a fondo del núcleo familiar y su relacionamiento al interior del hogar.

Recordemos que en el proceso formativo del estudiante interviene la comunidad educativa que está integrada por la familia, la institución educativa y el Estado; por lo tanto, el trabajo debe ser mancomunado y dirigido a un único objetivo $\mathrm{y}$ es el del desarrollo integral y dimensional del estudiante. Se deben formar personas, en suma, capaces de manejar y controlar las emociones con proyectos de vida definidos que enriquezcan la interacción social y la sana convivencia. 


\section{BIBLIOGRAFÍA}

- Akl, P. y Docal, M. (2014). Caracterización de la violencia intrafamiliar en el municipio de Villapinzón. Informe de investigación inédito. Chía, Colombia: Universidad de la Sabana.

- Secretaría de Gobierno de la Alcaldía de Bogotá, Corporación Excelencia de la Justicia y SUR (2013 c). Caracterización de la demanda. Bogotá, Colombia: Alcaldía Mayor.

- Allen, B., Chávez, R., Lazcano, E., Rivera L. y Rodríguez, G. (2006). Violencia durante el noviazgo: depresión y conductas de riesgo en estudiantes femeninas (12-24 años). México: Salud pública de México, 48, 288-296.

- Amador, R., Arias, J. y Amador, R. (2012). Tipos de violencia en el noviazgo: estudiantes universitarias de la UAEM, Zumpango. Revista electrónica de Psicología Iztacala, 15(1), 150-171.

- Barrientos, J., Molina, C. y Salinas D. (2013 a). Las causas de la violencia intrafamiliar en Medellín. Perfil de coyuntura económica, 22, 99-112.

- Bustamante, S., Gómez, M., Figueroa, Y. y Gómez, K. (2008 b). Encuesta de violencia en el noviazgo en cinco instituciones educativasdenivelsuperior del municipio de Oaxaca de Juárez. Oaxaca, México: Instituto Municipal de las Mujeres. Recuperado de: http://municipiodeoaxaca.gob.mx/ transparencia/archivos/Articulo\%209/ XXI\%20INFORMACION\%20RELEVANTE/ ESTUDIOS\%20E\%20INVESTIGACIONES/ ENCUESTA $\% 20$ DE $\% 2$ OVIOLENCIA $\% 20$ EN\%20EL\%20NOVIAZGO.docx.

- Chaux, E. (2003 a). Agresión reactiva, agresión instrumental y el ciclo de la violencia. Revista de estudios sociales, 16, 47-58.
- Cruz, B., Lettau, P. y Montoya, A. (2013 b). Porque te quiero... Una mirada a la violencia basada en género en las relaciones de noviazgo en la ciudad de Cartagena de Indias. Cartagena, Colombia: Revista Juris, 16, 181-200.

- González, M. (2009). Violencia en las relaciones de noviazgo entre jóvenes y adolescentes de la comunidad de Madrid (tesis de doctorado). Universidad Complutense de Madrid, España.

- Instituto Nacional de Medicina Legal y Ciencias Forenses (2015). Forensis 2014: datos para la vida. Bogotá, Colombia: Imprenta Nacional.

- Organización Mundial de la Salud (2002). Informe Mundial sobre violencia y salud: resumen. Washington, D.C., Estados Unidos: Organización Panamericana de la Salud.

- Ortiz, E. (2003 b). Estudio de diagnóstico de la violencia intrafamiliar en la localidad de Usme. Bogotá, Colombia: Colección de investigaciones del ICBF.

- Rey, C. (2008 a). Prevalencia, factores de riesgo y problemáticas asociadas con la violencia en el noviazgo: una revisión de la literatura.: Avances en Psicología Latinoamericana, 26(2), 227-241.

- Salas, L. (2005). Transmisión intergeneracional de la violencia intrafamiliar: evidencia para las familias colombianas. Centro de Estudios sobre Desarrollo Económica, CEDE, Universidad de los Andes. Recuperado de: https:// economia.uniandes.edu.co//components/ com_booklibrary/ebooks/d2005-47.pdf

- Velázquez, G. (2011). La violencia durante el noviazgo de adolescentes. Elementos: ciencia y cultura, 19(82), pp. 39-44. 
